\title{
DESENVOLVIMENTO DE DISPOSITIVO DE QUITOSANA E XANTANA PARA A LIBERAÇÃO TÓPICA OU EM TECIDOS MOLES DE INDOMETACINA
}

\author{
SELMA. A. LOPES $^{1}$, ITIARA G.VEIGA ${ }^{1,2}$, ÂNGELA M. MORAES $^{1}$ \\ ${ }^{1}$ Universidade Estadual de Campinas, Faculdade de Engenharia Química, Departamento de \\ Engenharia de Materiais e de Bioprocessos, Campinas, SP \\ ${ }^{2}$ Universidade Federal do Rio Grande, Escola de Química e Alimentos, Campus Santo \\ Antônio da Patrulha, RS \\ E-mail para contato: ammoraes@feq.unicamp.br
}

\begin{abstract}
Resumo - Dispositivos que possibilitam a liberação controlada de fármacos são de grande relevância, podendo contribuir para a melhoria da saúde humana. Neste trabalho produziu-se uma matriz que combina quitosana e xantana com indometacina, um fármaco com ação anti-inflamatória, analgésica e antipirética comumente administrado por via oral, visando seu uso na terapia de lesões de pele e cartilagem. A incorporação da indometacina foi feita por adição direta aos polissacarídeos em suspensão aquosa, sendo o material caracterizado quanto ao aspecto, espessura, eficiência de incorporação e à cinética de liberação do fármaco. Observou-se que as membranas, translúcidas e esbranquiçadas, com espessura de 0,09 a 0,10 mm, incorporam mais de 90\% do fármaco adicionado. Parte do fármaco se dispersa na matriz e parte deposita-se em sua superfície; sua total liberação ocorre em até $24 \mathrm{~h}$. A caracterização adicional por TGA e FTIR/ATR indicou interações entre as espécies e boa estabilidade térmica do material.
\end{abstract}

\section{INTRODUÇÃO}

O desenvolvimento de dispositivos de liberação controlada de fármacos tem promovido crescente interesse nas últimas décadas, contribuindo para o tratamento de variados tipos de lesões. Para isto, podem ser utilizados biopolímeros como a quitosana (Q) e a xantana (X), ambos capazes de formar complexos polieletrólitos (PECs) moldáveis na forma de membranas (Berger et al., 2004).

A quitosana é um polissacarídeo derivado da desacetilação alcalina da quitina. Em condições apropriadas de $\mathrm{pH}$, seus grupamentos amina possibilitam sua ligação a materiais com cargas negativas, como outros polissacarídeos, enzimas e células. Possui boas propriedades biológicas, como biodegradabilidade e biocompatibilidade, sendo atóxica e bioativa (Hein et al., 2008). 
A xantana é um polímero atóxico e estável em uma vasta gama de condições de temperatura e pH, sendo utilizada em indústrias alimentícias, farmacêuticas e cosméticas como emulsionante e estabilizador de suspensões. Pode ser processada sob a forma de géis, filmes e membranas (Bellini et al., 2012) e, por apresentar em sua estrutura grupos carboxila, em determinadas condições de $\mathrm{pH}$ pode interagir com moléculas positivamente carregadas, como a quitosana.

A incorporação do fármaco indometacina em dispositivos de Q-X tem bom potencial de aplicação no tratamento de lesões de pele e em tecidos moles, como cartilagens. Este composto tem ação anti-inflamatória, antipirética e analgésica, sendo praticamente insolúvel em água e pouco solúvel em meio ácido (Florey, 1984).

Neste contexto, neste trabalho teve-se por objetivo incorporar indometacina em membranas de Q-X por adição durante a produção da matriz polimérica, caracterizar os dispositivos obtidos e compará-los a formulações não contendo o fármaco.

\section{MATERIAIS E MÉTODOS}

\subsection{Materiais}

Para a obtenção das membranas, foram utilizados os seguintes reagentes de qualidade analítica certificada: quitosana obtida da carapaça de camarão com grau de acetilação mínimo de 75\% (lote: 061M0046V), xantana (lote: 056K0007), indometacina (lote: BCBC9386) da Sigma-Aldrich Co., ácido acético glacial da Synth e água destilada e deionizada em sistema Milli-Q da Millipore.

\subsection{Métodos}

Preparação das membranas de quitosana e xantana: os dispositivos foram preparados de acordo com o método descrito por Veiga e Moraes (2012). Para isto, uma alíquota de $90 \mathrm{~mL}$ de solução de quitosana a $0,5 \%(\mathrm{~m} / \mathrm{v})$ em solução de ácido acético a $2 \%(\mathrm{v} / \mathrm{v})$ foi adicionada através de bomba peristáltica Gilson (Miniplus 3), na vazão de $300 \mathrm{~mL} / \mathrm{h}$, em $90 \mathrm{~mL}$ de solução aquosa de xantana a $0,5 \%(\mathrm{~m} / \mathrm{v})$ em um reator de aço inoxidável encamisado com diâmetro interno de $9 \mathrm{~cm}$ e capacidade de $700 \mathrm{~mL}$. A temperatura foi mantida, durante todo o processo, a $25^{\circ} \mathrm{C}$ utilizando-se um banho termostático Quimis. Durante o gotejamento, o sistema foi mantido sob agitação constante de $1000 \mathrm{rpm}$ através de agitador mecânico Quimis provido de hélice do tipo naval e raio de $2,5 \mathrm{~cm}$. A incorporação de indometacina foi realizada por adição direta do fármaco na forma de pó logo após a etapa de mistura completa de quitosana à xantana, na proporção de $20 \mathrm{mg}$ de indometacina por grama de sólidos da mistura de polissacarídeos, sob agitação de $1000 \mathrm{rpm}$ por 1 minuto. A seguir, a mistura polimérica obtida foi desaerada em bomba de vácuo Quimis por $2 \mathrm{~h}$ e, em seguida, foi transferida para uma placa de Petri de poliestireno de $15 \mathrm{~cm}$ e seca a $37^{\circ} \mathrm{C}$ em estufa com circulação de ar, por $24 \mathrm{~h}$, sobre uma plataforma giratória com diâmetro de $25 \mathrm{~cm}$ operando a $3 \mathrm{rpm}$. Após a secagem, a membrana foi imersa por duas vezes em $200 \mathrm{~mL}$ de água deionizada por 30 minutos, para a remoção do ácido acético residual. As membranas foram 
então secas novamente a $37^{\circ} \mathrm{C}$ por $24 \mathrm{~h}$ e armazenadas em dessecador.

Aspecto e morfologia: as membranas foram inspecionadas a olho nu quanto ao aspecto e fotografadas com uma câmara digital Canon (Power Shot A410). As amostras foram também analisadas por microscopia eletrônica de varredura (MEV) (microscópio Leo 440i), quanto às características morfológicas da superfície e da secção transversal. Para a análise por MEV, as amostras $\left(2 \times 1 \mathrm{~cm}^{2}\right)$ foram criofraturadas, colocadas em suporte adequado, e metalizadas no equipamento mini Sputter coater (SC 7620), depositando-se uma camada de ouro (92 Å).

Espessura: esta propriedade foi determinada pela utilização de micrômetro (Digimess), através de medições em sete diferentes pontos ao longo da extensão da membrana. Os resultados foram expressos como média das medidas.

Análise da eficiência de incorporação do fármaco (E): a eficiência do processo foi estimada após a extração do fármaco das amostras em solução de tampão fosfato salino (PBS) a pH 7,4 por $24 \mathrm{~h}$ e a determinação de sua concentração por espectrofotometria de absorção de luz a 319 nm, utilizando-se a Equação 1:

$$
\varepsilon=\frac{(\text { massa final de fármaco } / \text { massa de polímeros })}{(\text { massa inicial de fármaco } / \text { massa de polímeros })} \times 100
$$

Avaliação da cinética de liberação do fármaco: para este ensaio, as amostras $\left(1 \mathrm{x} 1 \mathrm{~cm}^{2}\right.$, de massa conhecida) foram imersas em cubetas de quartzo contendo $4 \mathrm{~mL}$ de PBS (pH 7,4), à temperatura de $37^{\circ} \mathrm{C}$ e agitação de $100 \mathrm{rpm}$. A fase aquosa foi periodicamente analisada quanto à concentração de indometacina por espectrofotometria de absorção de luz a $319 \mathrm{~nm}$.

Análise termogravimétrica (TGA): esta análise foi realizada através das medidas de perda de massa das amostras com massa inicial de $6 \mathrm{mg}$ utilizando um analisador termogravimétrico (TA Instruments, Q500, USA). Os experimentos foram conduzidos a partir da temperatura ambiente até $600^{\circ} \mathrm{C}$, com taxa de aquecimento de $10^{\circ} \mathrm{C} / \mathrm{min}$ e atmosfera de nitrogênio (vazão de $40 \mathrm{~mL} / \mathrm{min}$ ).

Espectroscopia no infravermelho por transformada de Fourier: os espectros foram obtidos em um espectrofotômetro FTIR/ATR Nicolet 6700 da marca ThermoScientific. A varredura de absorção foi realizada de 675 a $4000 \mathrm{~cm}^{-1}$, com resolução de $4 \mathrm{~cm}^{-1}$ e 64 varreduras acumuladas.

\section{RESULTADOS E DISCUSSÃO}

O aspecto a olho nu das membranas contendo ou não indometacina pode ser observado na Figura 1, enquanto na Figura 2 mostra-se a morfologia das mesmas analisadas por MEV. Nota-se que as membranas são relativamente homogêneas, apresentando fibras em sua superfície (provavelmente dos PECs). Percebe-se também a presença de precipitados esbranquiçados (provavelmente a indometacina) depositados na superfície e entre as lamelas nas amostras contendo o fármaco. 

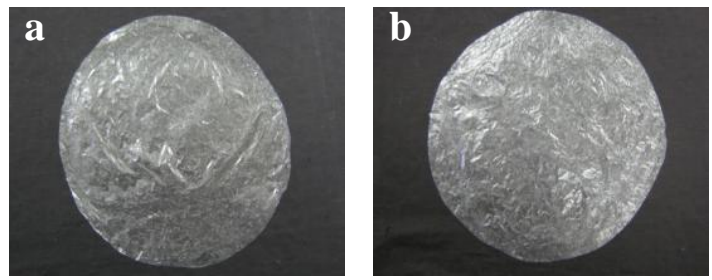

Figura 1 - Aspecto das membranas de quitosana e xantana preparadas (a) na ausência e (b) na presença de $20 \%$ de indometacina.
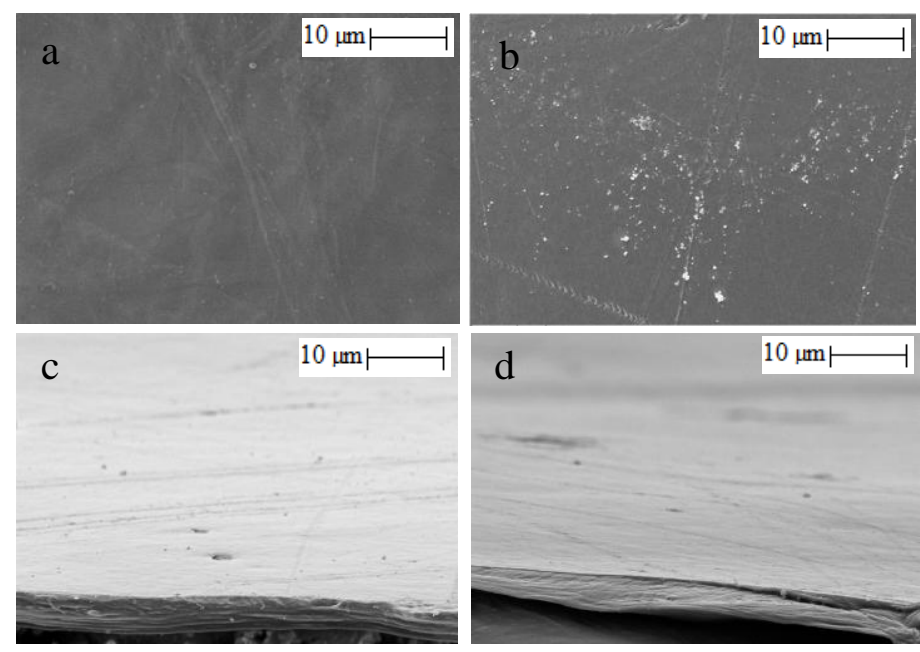

Figura 2 - Superfícies das membranas de Q-X preparadas (a) na ausência e (b) na presença de $20 \%$ de indometacina e secção transversal das membranas de Q-X preparadas (c) na ausência e (d) na presença de $20 \%$ de indometacina.

Os dados de espessura média das amostras e de eficiência de incorporação do fármaco estão mostrados na Tabela 1. Observa-se que a espessura das membranas praticamente não é afetada pela adição do fármaco. Nota-se também que a eficiência de incorporação da indometacina é muito alta, próxima de $99 \%$. A pequena perda observada provavelmente pode ter ocorrido durante a etapa de lavagem da membrana em água.

Os resultados obtidos na análise da cinética de liberação do fármaco incorporado nas amostras por exposição à solução de PBS por 24 h estão indicados na Figura 3. Observa-se a ocorrência de rápida liberação nas primeiras 2 horas, atingindo-se o máximo de liberação em $5 \mathrm{~h}$ de exposição. Atribui-se tal comportamento ao rápido intumescimento da membrana em PBS (Veiga e Moraes, 2012), o que facilita a liberação do fármaco presente no interior da matriz e também à grande quantidade de fármaco depositada na superfície da amostra, que se solubiliza ainda mais facilmente.

Os resultados da análise por TGA estão apresentados na Figura 4 e sumarizados na Tabela 2. O primeiro evento térmico identificado para os biopolímeros separados e complexados nas membranas, na faixa de 60 a $80^{\circ} \mathrm{C}$, refere-se a perdas de massa em torno de 
$6 \%$ a $12 \%$, possivelmente relacionadas à evaporação de água presente nas amostras (Fahmy e Fouda, 2008). Como o fármaco não é higroscópico, não se observa perda de água para a indometacina nesta faixa de temperatura (Marini et al., 2003).

Tabela 1 - Espessura média e eficiência de incorporação de indometacina nas membranas.

\begin{tabular}{cccc}
\hline $\begin{array}{c}\text { Razão inicial } \\
\text { fármaco/polímero }(\mathbf{m g} / \mathbf{g})\end{array}$ & $\begin{array}{c}\text { Espessura } \\
(\boldsymbol{\mu} \mathbf{m})\end{array}$ & $\begin{array}{c}\text { Razão final } \\
\text { fármaco/polímero }(\mathbf{m g} / \mathbf{g})\end{array}$ & $\begin{array}{c}\text { Eficiência de } \\
\text { incorporação }(\boldsymbol{\%})\end{array}$ \\
\hline 0 & $95 \pm 16$ & - & 0 \\
20 & $94 \pm 15$ & $19,7 \pm 4,1$ & 98,5 \\
\hline
\end{tabular}

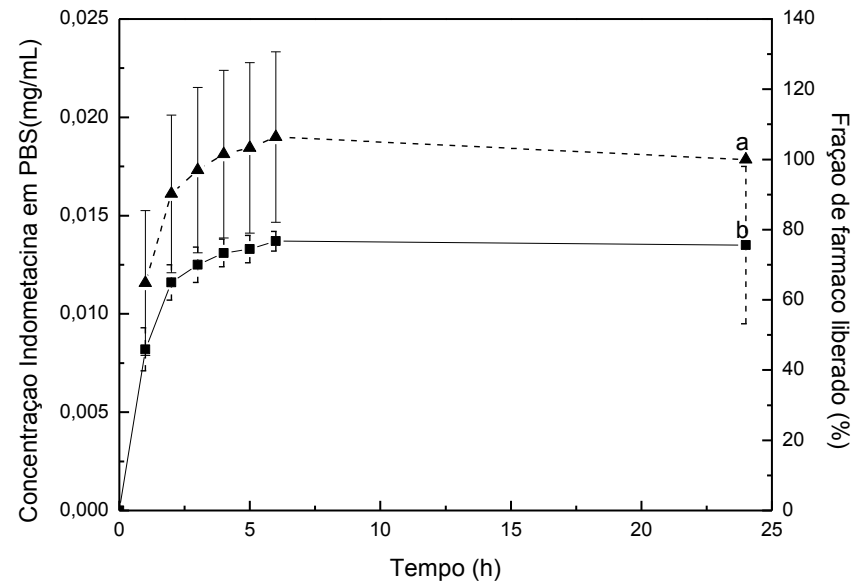

Figura 3 - Cinética de liberação em solução de PBS do fármaco incorporado nas membranas de quitosana e xantana ao longo de $24 \mathrm{~h}$.

Nos eventos com picos entre $100^{\circ} \mathrm{C}$ e $280^{\circ} \mathrm{C}$ são observadas perdas de massa crescentes para os polissacarídeos isolados, entretanto, para as membranas as perdas foram bem menores nesta faixa de temperatura, observando-se perdas de 18 e $15 \%$ para amostras contendo ou não o fármaco, respectivamente com picos em $273^{\circ} \mathrm{C}$ e $268^{\circ} \mathrm{C}$. Tais resultados indicam a ocorrência de interações entre os compostos envolvidos, no caso interações iônicas entre as aminas da $\mathrm{Q}$ e as carboxilas da $\mathrm{X}$ e entre as aminas da Q e a carboxila da indometacina, que tem pKa de 4,5, de acordo com Florey (1984), que contribuem para a diminuição da temperatura de degradação em comparação às dos polissacarídeos isolados.

Em temperaturas acima de $280^{\circ} \mathrm{C}$, observa-se degradação térmica acentuada da quitosana, da xantana e do fármaco isolados e combinados entre si, com picos identificados na faixa de $312^{\circ} \mathrm{C}$ a $340^{\circ} \mathrm{C}$. A presença do fármaco, que tem pico de degradação térmica mais alto que os polissacarídeos, na membrana fez com que se tivesse degradação um pouco menor no dispositivo, a uma temperatura com pico ligeiramente superior ao observado para a formulação livre do fármaco. 

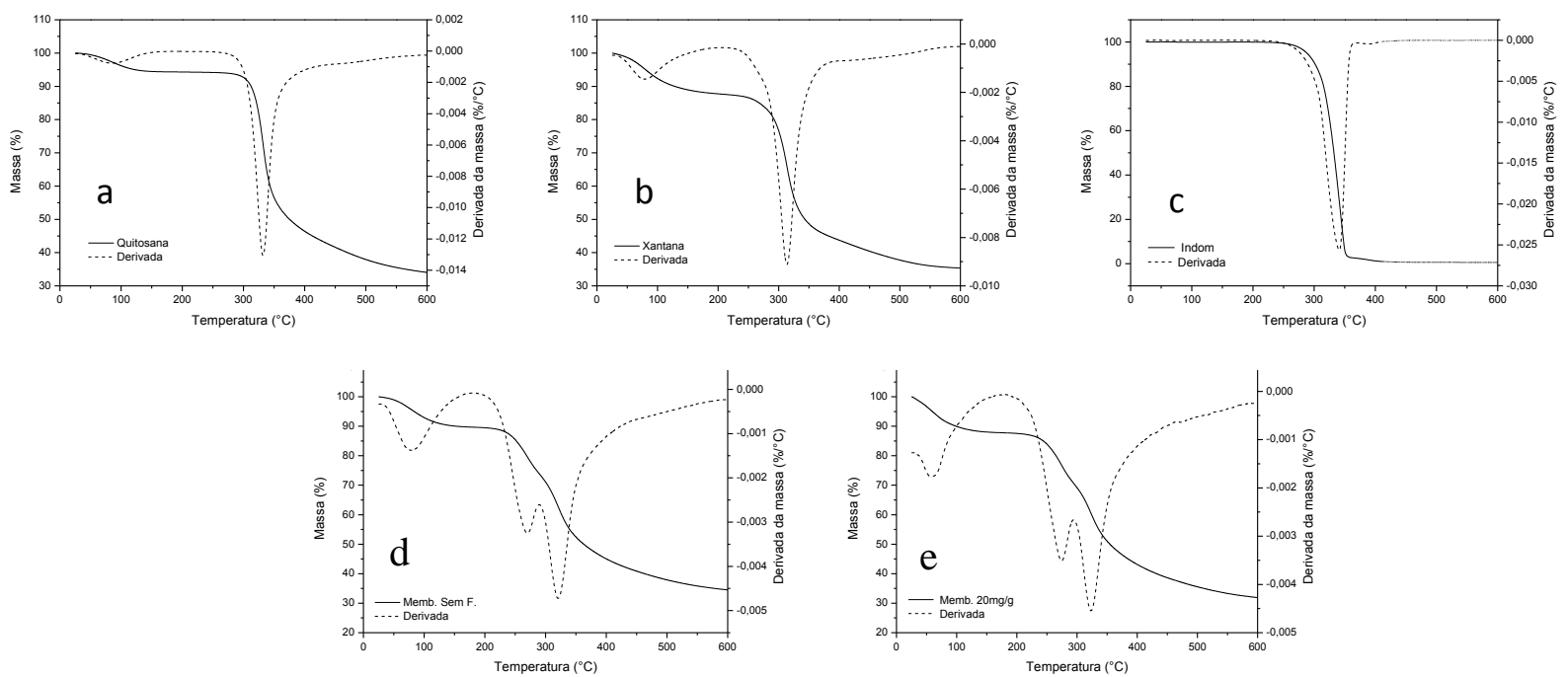

Figura 4 - Termogramas da (a) quitosana, (b) xantana e (c) indometacina isoladas e das membranas de quitosana e xantana preparadas (d) na ausência e (e) na presença de $20 \%$ de indometacina.

Tabela 2 - Sumário dos principais eventos de degradação térmica (observados em diferentes faixas de temperatura) dos biopolímeros e do fármaco isolado e das membranas contendo ou não indometacina.

\begin{tabular}{lcccccc}
\hline \multirow{2}{*}{ Composição da amostra } & \multicolumn{2}{c}{ Evento 1 } & \multicolumn{2}{c}{ Evento 2 } & \multicolumn{2}{c}{ Evento 3 } \\
\cline { 2 - 7 } & $\begin{array}{c}\text { Pico de T } \\
\left({ }^{\circ} \mathbf{C}\right)\end{array}$ & $\begin{array}{c}\text { Perda de } \\
\text { massa }(\%)\end{array}$ & $\begin{array}{c}\text { Pico de T } \\
\left({ }^{\circ} \mathbf{C}\right)\end{array}$ & $\begin{array}{c}\text { Perda de } \\
\text { massa(\%) }(\%)\end{array}$ & $\begin{array}{c}\text { Pico de T } \\
\left({ }^{\circ} \mathbf{C}\right)\end{array}$ & $\begin{array}{c}\text { Perda de } \\
\text { massa(\%) }\end{array}$ \\
\hline Quitosana & 80 & 6 & - & - & 331 & 60 \\
Xantana & 79 & 12 & - & - & 312 & 52 \\
Indometacina & - & - & - & - & 340 & 99 \\
Membrana de Q-X & 80 & 10 & 268 & 15 & 320 & 40 \\
Membrana de Q-X com & 60 & 12 & 273 & 18 & 324 & 38 \\
indometacina & & & & &
\end{tabular}

Os espectros de FTIR/ATR obtidos pela análise das membranas, dos polissacarídeos e do fármaco isolados são apresentados na Figura 5. No caso dos biopolímeros isolados, é possível observar regiões de absorção típicas dos polissacarídeos, como bandas em torno de $1000 \mathrm{~cm}^{-1}$ (ligações C-O e C-C), 3700 e $3000 \mathrm{~cm}^{-1}$ (O-H) e 3000 e $2800 \mathrm{~cm}^{-1}$ (C-H) (Pawlak e Mucha, 2003). Para a quitosana isolada, nota-se as bandas características com picos em 1641 e $1556 \mathrm{~cm}^{-1}$ relativas à ligação $(\mathrm{C}=\mathrm{O})$ dos grupos amida acetilados e $\mathrm{N}-\mathrm{H}_{2}$ dos grupos amina, respectivamente. A xantana, quando isolada, apresenta picos em 1739 e $1610 \mathrm{~cm}^{-1}$ provavelmente relativos aos grupos carboxílicos ionizados $\left(-\mathrm{C}=\mathrm{OO}^{-}\right)$e de éster insaturado, típico dos grupos acetato e piruvato (Popa et al., 2010). A indometacina apresenta bandas no 
intervalo de 3400 a $2500 \mathrm{~cm}^{-1}$ relativas ao estiramento do grupo aromático $(\mathrm{C}-\mathrm{H})$ e do grupo ácido carboxílico (O-H), entre 1711 e $1690 \mathrm{~cm}^{-1}$ referentes ao estiramento da ligação $\mathrm{C}=\mathrm{O}$ e ao estiramento do $\mathrm{C}=\mathrm{C}$ em $1587 \mathrm{~cm}^{-1}$ ). Além destes, há picos em $1477 \mathrm{~cm}^{-1}$ referentes à deformação do $\mathrm{O}-\mathrm{CH}_{3}$, em $1232 \mathrm{~cm}^{-1}$ possivelmente relativos ao estiramento do C-O em conjunto com a deformação do $\mathrm{O}-\mathrm{H}$, em $929 \mathrm{~cm}^{-1}$ relativo à deformação planar do grupo $\mathrm{O}-\mathrm{H}$ carboxílico e na região de 904 a $690 \mathrm{~cm}^{-1}$ referentes à deformação planar da ligação C-H de grupos aromáticos substituídos (Florey, 1994).

No espectro da membrana de Q-X na ausência de fármaco observa-se a presença de uma banda de absorção entre 1709 e $1562 \mathrm{~cm}^{-1}$. A formação desta banda se deve à justaposição dos picos referentes aos grupos amino da quitosana, e aos grupos carboxílicos da xantana, o que sugere a interação iônica entre estes grupos (Popa et al., 2010).

Observa-se também na Figura 6 que as membranas de Q-X contendo ou não o fármaco possuem espectros com perfis muito semelhantes entre si. Logo, não foi possível identificar pela análise de FTIR/ATR, a presença do fármaco nas amostras. Porém, pela análise da morfologia obtida por MEV, observa-se claramente a presença de precipitados depositados na superfície das membranas, o que sugere a sobreposição dos picos referentes ao fármaco e aos polissacarídeos, resultando no encobrimento dos picos associados à indometacina.

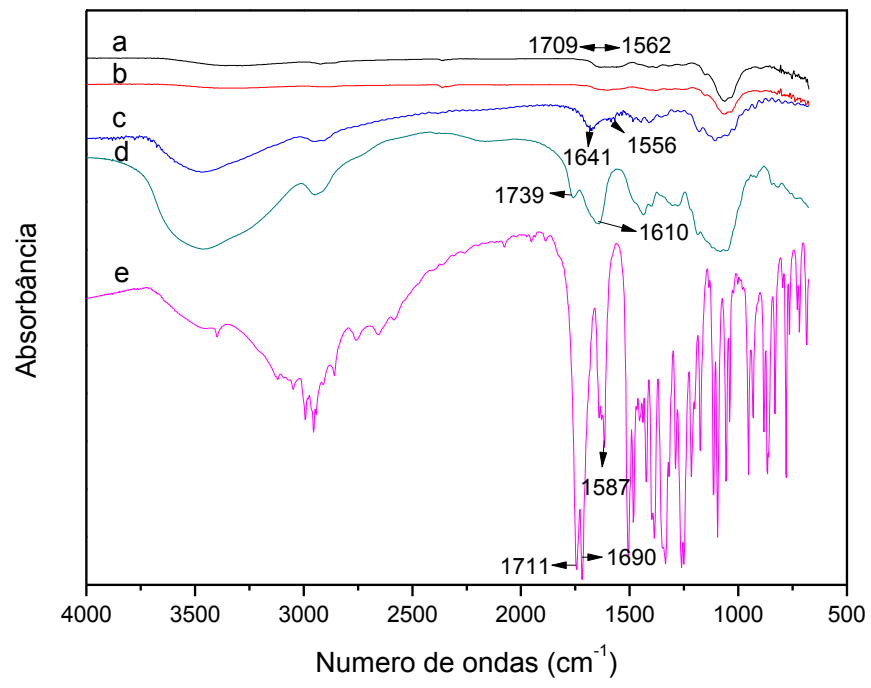

Figura 5. Espectro FTIR-ATR das membranas de Q-X (a) na ausência e na (b) presença de $20 \%$ de indometacina, e dos compostos isolados (c) quitosana, (d) xantana e (e) indometacina.

\section{CONCLUSÃO}

Os resultados obtidos para a incorporação de indometacina em dispositivos de quitosana 
e xantana pelo método de adição à mistura polissacarídica indicam que esta metodologia é eficaz, uma vez que o material obtido tem aspecto adequado e a eficiência de incorporação é muito elevada, próxima de $100 \%$. As membranas não sofrem grande alteração na espessura em decorrência da incorporação da indometacina. A maior parte do fármaco incorporado é liberada dentro de $2 \mathrm{~h}$ e, em $5 \mathrm{~h}$ já se atinge a liberação máxima de $100 \%$. A razão de cerca de $20 \mathrm{mg}$ de fármaco incorporado por grama de biopolímero atende às necessidades de aplicação in vivo desejadas. As caracterizações por TGA e FTIR/ATR mostram que as membranas apresentaram alta estabilidade térmica e que ocorrem interações iônicas entre as cadeias dos biopolímeros e o fármaco. Portanto, as membranas obtidas têm propriedades promissoras para a aplicação como curativos em lesões de pele e em tecidos moles.

\section{REFERÊNCIAS}

BELLINI, M.; PIRES, A.L.R.; VASCONCELOS, M.O.; MORARES, A.M. Comparison of the properties of compacted and porous lamellar chitosan-xanthan membranes as dressings and scaffolds for the treatment of skin lesions. J. Appl. Polym. Sci., v. 125 (S2), p. E421-E431, 2012.

BERGER, J.; REIST, M.; MAYER, J. M.; FELT, O.; GUMY, R. Structure and interactions in chitosan hydrogels formed by complexation or aggregation for biomedical applications. Eur. J. Pharm. Biopharm., v. 57, p. 35-52, 2004.

FAHMY, H. M.; FOUDA, M.G. Crosslinking of alginic acid/chitosan matrices using polycarboxylic acids and their utilization for sodium diclofenac release. Carbohyd. Polym., v. 73, p. 606-611, 2008.

FLOREY, K. Analytical profiles of drug substances, v. 13, p. 211-235, 1984.

HEIN, S.; WANG, K.; STEVENS, W. F.; KJEMS, J. Chitosan composites for biomedical applications: status, challenges and perspectives. Mater. Sci. Tech. Ser., v. 24, p.10531061, 2008.

MARINI, A., BERBENNI, V., MOIOLI, S., BRUNI, G., COFRACESCO, P. MARGHERITIS, C., VILLA, M. Drug-excipient compatibility studies by physicochemical techniques. The case of indomethacin. J. Therm. Anal. Calorim., v. 13, p. 529-545, 2003.

PAWLAK, A., MUCHA, M. Thermogravimetric and FTIR studies of chitosan blends. Thermochim. Acta., v. 396, p. 153-166, 2003.

POPA, N., NOVAC, O., PROFIRE, O., LUPUSORU, C. E., POPA, M.I. Hydrogels based on chitosan-xanthan for controlled release of theophylline. J. Mater. Sci: Mater. Med., v. 21, p.1241-1248, 2010.

VEIGA, I. G.; MORAES, A.M. Study of the swelling and stability properties of chitosanxanthan membranes. J. Appl. Polym. Sci., v. 124, p. E154-E160, 2012.

AGRADECIMENTOS: À CAPES e ao CNPq, pelo apoio financeiro. 